\title{
ON A CERTAIN CLASS OF SYMMETRIC HYPERSURFACES
}

DARRELL R. SHREVE

In the literature of algebraic geometry, relatively little is written on hypersurfaces $H$ of order $n$ and of $r-1$ dimensions in $S_{r}$, invariant under the symmetric permutation group $G$ on $r+2$ homogeneous coordinates whose sum is zero. Surfaces in $S_{3}$ invariant under the symmetric $G_{120}$ have been studied by Emch [1]; the Clebsch diagonal surface has been discussed by Clebsch [2], Eckardt [3], and Ciani [4, $5,6]$. The Segre cubic variety in $S_{4}$ has been investigated by Segre [8]; Ciani [7] has developed properties of loci in $S_{4}$ invariant under the $G_{720}$.

It is well known that the equation of such a hypersurface $H$ is exexpressible uniquely in terms of the elementary symmetric functions $p_{i}$ (of order $i$ in $x_{1}, \cdots, x_{r+2}$ ), or in terms of the $\Sigma$ functions $\Sigma_{n}=\sum_{i=1}^{r+2} x_{i}{ }^{n}$. With the condition $\Sigma_{1}=0$, any $H$ is a member of the linear system

$$
\sum_{i=1}^{N} C_{i} \Sigma_{2}^{a} \Sigma_{3}^{b} \cdots \Sigma_{r+2}^{d}=0
$$

where $N$ is the number of nonnegative solutions of the Diophantine equation

$$
2 a+3 b+\cdots+(r+2) d=0 .
$$

It follows immediately that there is a unique hyperquadric $H_{2}, \Sigma_{2}=0$ or $p_{2}=0$, a unique cubic $H_{3}, \Sigma_{3}=0$ or $p_{3}=0$, a pencil of quartics $H_{4}$, $\lambda \Sigma_{4}+\mu \Sigma_{2}{ }^{2}=0$, a pencil of quintics $H_{5}, \lambda \Sigma_{5}+\mu \Sigma_{2} \Sigma_{3}=0$, and so on, in $S_{r}$ invariant under $G$.

Since there are no real points on $\Sigma_{n}=0$ if $n$ is even, values of $n$ will be restricted to odd positive integers throughout the remainder of this paper.

Emch [1] has shown that the equation of any surface of odd order in $S_{3}$, invariant under the symmetric $G_{120}$ on $5 x$ 's whose sum is zero necessarily has the form $A p_{3}+B p_{5}=0$, which is equivalent to $\lambda \Sigma_{3}+\mu \Sigma_{5}=0$. The obvious generalization of this statement is that any $H$ of order $n$ necessarily is a member of the linear system

$$
A \Sigma_{3}+B \Sigma_{5}+\cdots+D \Sigma_{r+2}=0 ;
$$

if $r=2 k+1$, and in case $r=2 k$, of the system

$$
A \Sigma_{3}+B \Sigma_{5}+\cdots+C \Sigma_{r+1}=0 .
$$


If $r=2 k+1$, all $H$ of (3) contain the base $\Sigma_{3}=\Sigma_{5}=\cdots=\Sigma_{r+2}=0$. This base is of order $\alpha=(r+2) \cdot r ! / 2^{k} \cdot k$ ! and of dimension $k$, consisting of the $\alpha$ subspaces into which the $S_{k}\left[x_{1}+x_{2}=0, x_{3}+x_{4}=0, \cdots\right.$, $\left.x_{i}+x_{r+1}=0, x_{r+2}=0\right]$ is carried by the substitutions of $G$. Each $S_{k}$ is invariant under a subgroup of $G$ of order $2^{k+1} \cdot(k+1)$ !.

If $r=2 k$, all $H$ of (4) contain the base $\Sigma_{3}=\Sigma_{5}=\cdots=\Sigma_{r+1}=0$, of order $\beta=(r+1) ! / 2^{k} \cdot k$ ! and of dimension $k$, consisting of the $\beta$ subspaces into which the substitutions of $G$ carry the $S_{k}\left[x_{1}+x_{2}=0\right.$, $\left.x_{3}+x_{4}=0, \cdots, x_{r+1}+x_{r+2}=0\right]$; each $S_{k}$ is invariant under a subgroup of $G$ of order $2^{k+1} \cdot(k+1)$ !.

The hypersurfaces $\Sigma_{n}=0$ have as double points only those points whose coordinates are proportional to $(n-1)$ th roots of unity, since at a double point the $r+1$ partial derivatives $\partial \Sigma_{n} / \partial x_{i}=n x_{i}{ }^{n-1}-n x_{r+2}^{n-1}$, $i=1,2, \cdots, r+1$, must vanish (with the dependence of $x_{r+2}$ expressed by $\left.\Sigma_{1}=0\right)$. It follows immediately that $\Sigma_{n}=0$ has $C_{r+1, k}$ real double points if $r=2 k$, and has no real double points if $r=2 k+1$. Imaginary double points of $\Sigma_{n}=0$ will occur whenever there is a set of $r+2(n-1)$ th roots of unity, not all real, whose sum is zero.

If $r=2 k+1$, then none of the $\alpha$ subspaces $S_{k}$ passes through a double point of $\Sigma_{n}=0$, since at a double point no $x_{i}=0$.

If $r=2 k$, then in each of the $\beta$ subspaces $S_{k}$ there are $(n-1)^{k}$ double points of $\Sigma_{n}=0$, of which exactly $2^{k}$ are real; through each of the real double points pass $(k+1)$ ! subspaces $S_{k}$. If $r=2 k$, and $2 k+1$ is a prime, then the $\beta$ subspaces $S_{k}$ divide into $(r-1)$ ! sets of $(r+1)$ tuples, each $(r+1)$-tuple being transformable into another $(r+1)$ tuple by a cyclic substitution of $G$ of period $r+1$. (This is a generalization of the 6 quintuples of planes on the Segre cubic variety.)

The hypersurfaces $\Sigma_{n}=0$ can contain no points of multiplicity greater than two, since not all the partial derivatives of higher order vanish at any point.

Eckardt [3] has given an admirable synthetic and analytic discussion of the properties of an Eckardt point of a surface in $S_{3}$. The analytic generalization is immediate. Let a generalized Eckardt point $E$ of a hypersurface $F$, of order $m>2$ and of $r-1$ dimensions in $S_{r}$, be a simple point of $F$ such that the hyperplane $T$ tangent to $F$ in $E$ intersects $F$ in a hypercone with a vertex at $E$. We may so choose the polylateral of reference in $S_{r}$ that $E$ is $(1,0, \cdots, 0)$, and $T$ is $x_{2}=0$. Then the equation of $F$ necessarily has the form

$$
x_{2} x_{1}^{m-1}+a_{1} x_{2} x_{1}^{m-2}+\cdots+a_{m-2} x_{2} x_{1}+a_{m}=0
$$

where $a_{i}$ is a form of order $i$ in $x_{2}, x_{3}, \cdots, x_{r+1}$.

It follows immediately that the $i$ th polar of $E$ reduces into $T$ 
$\left(x_{2}=0\right)$ and a hypersurface of order $m-i-1$ which does not pass through $E$.

Conversely, if the polar of a point $P$ with respect to a hypersurface $F$ of order $m$ reduces into a hyperplane $\pi$ passing through $P$ and a hypersurface of order $m-2$ not passing through $P$, then if $F$ does not reduce into $\pi$ and a hypersurface of order $m-1, P$ is a generalized Eckardt point of $F$ and $\pi$ is tangent to $F$ at the point $P$.

On each hypersurface $\Sigma_{n}=0$ in $S_{r}$ there are $C_{r+2,2}$ Eckardt points $E_{i j}\left(x_{i}=-x_{j} \neq 0 ; x_{s}=0, s \neq i, j\right)$, with the hyperplane $x_{i}+x_{j}=0$ tangent to $\Sigma_{n}=0$ at $E_{i j}$. The polar of $E_{i j}$ with respect to $\Sigma_{n}=0$ is $x_{i}^{n-1}-x_{j}^{n-1}=0$, which contains the hyperplanes $x_{i}+x_{j}=0$ and $x_{i}-x_{j}=0$. The latter is the axis of the perspectivity $(i j)$, with center at $E_{i j}$, under which $\Sigma_{n}=0$ and the polar of $E_{i j}$ are invariant. The $(n-1)$-fold locus $x_{i}=x_{j}=0$ of the polar of $E_{i j}$ contains $C_{r, 2}$ Eckardt points $E_{s t},(s, t \neq i, j)$.

If $r=2 k$, the hyperplane $x_{i}+x_{j}=0$ contains $C_{r, k}$ real double points of $\Sigma_{n}=0$, and $x_{i}-x_{i}=0$ contains the remaining $C_{r, k-1}$ real double points. Each real double point $D$ of $\Sigma_{n}=0$ is collinear with $(k+1)^{2}$ couples of points of $\Sigma_{n}=0$, each couple consisting of an $E_{i j}$ and the double point corresponding to $D$ under $(i j)$. In each of the $\beta$ subspaces $S_{k}$ on $\Sigma_{n}=0$ there are $k+1$ points $E_{i j}$, and through each $E_{i j}$ there pass $r ! / 2^{k} k$ ! subspaces $S_{k}$, while the three collinear points $E_{i j}, E_{i k}, E_{j k}$ do not lie in a common $S_{k}$ on $\Sigma_{n}=0$. An $S_{k}$ is the locus of points common to the $k+1$ hyperplanes tangent to $\Sigma_{n}$ at the $k+1$ points $E_{i j}$ in the $S_{k}$.

If $r=2 k+1$, through each $E_{i j}$ on $\Sigma_{n}=0$ pass $(r+1) ! / 2^{k} \cdot k$ ! subspaces $S_{k}$, with $k+1$ points $E_{i j}$ in each $S_{k}$.

The Eckardt point of a hypersurface $F$ is in general of multiplicity $r-1$ on the Hessian of $F$; the Eckardt points of $\Sigma_{n}=0$ are of multiplicity $(r-1)(n-2)$ on the Hessian of $\Sigma_{n}=0$, whose equation is $\sum_{i=1}^{r+2} 1 / x_{i}^{n-2}=0$.

\section{REFERENCES}

1. Arnold Emch, Some geometric applications of the symmetric collineation groups on five variables, Tôhoku Mathematical Journal, vol. 40 (1934), pp. 216-221.

2. A. Clebsch, Ueber die Anwendung der quadratischen Substitution auf die Gleichungen 5ten Grades und die geometrische Theorie des ebenen Fünfseits, Mathematische Annalen, vol. 4 (1871). See in particular pp. 331-340.

3. F. E. Eckardt, Ueber diejenigen Flächen dritten Grades, auf denen sich drei gerade Linien in einem Punkte schneiden, Mathematische Annalen, vol. 10 (1876), pp. 227-272.

4. Edgardo Ciani, Sulla superficie diagonale di Clebsch, Rendiconti della Accademia dei Lincei, vol. 7 , no. 6 . 
5. —-, Sul pentaedro complete, Rendiconti della Accademia dei Lincei, vol. 7, no. 5 .

6. - Sopra la configurazione del pentaedro, Rendiconti del Circolo Matematico di Palermo, vol. 21 (1906), pp. 322-341.

7. — Una interpretazione geometrica del gruppo totale di sostituzioni sopra sei elementi, Annali di Matematica Pura ed Applicata, 1909.

8. Corrado Segre, Sulla varietà cubica con dieci punti doppi dello spazio a quattro dimensioni, Atti della Accademia delle Scienze di Torino, vol. 22 (1886-1887), p. 791.

Purdue University

\section{CORRECTION TO "ON GREEN'S FUNCTIONS IN THE THEORY OF HEAT CONDUCTION IN SPHERICAL COORDINATES"*}

\section{A. N. LOWAN}

In the article entitled On Green's functions in the theory of heat conduction by H. S. Carslaw and J. C. Jaeger (this Bulletin, vol. 45 (1939), pp. 407-413), a misprint is noted in the expression for $G$ on page 133 of my article $O n$ the operational determination of two dimensional Green's functions in the theory of heat conduction (this Bulletin, vol. 44 (1938), pp. 125-133), the correct expression for $G$ being

$$
\begin{aligned}
G=u+v & =\frac{1}{4 \pi} \sum_{n=-\infty}^{\infty} \cos n\left(\theta-\theta_{0}\right) \int_{-\infty}^{\infty} \alpha e^{-k \alpha^{2} t} \frac{H_{n}^{(1)}\left(\alpha r_{0}\right)}{U_{n}(a \alpha)} \\
& \cdot\left\{J_{n}(\alpha r) U_{n}(\alpha a)-H_{n}^{(1)}(\alpha r)\left[\alpha \frac{d}{d z} J_{n}(z)+h J_{n}(z)\right]_{z=\alpha a}\right\} d \alpha,
\end{aligned}
$$

where

$$
U_{n}(\alpha a)=\left[\alpha \frac{d}{d z} H_{n}^{(1)}(z)+h H_{n}^{(1)}(z)\right]_{z=\alpha a} .
$$

When this correct expression is employed, formula (20), page 313, of the present paper becomes

$$
\begin{aligned}
& G\left(r, \theta, \phi, t ; r_{0}, \theta_{0}, \phi_{0}\right)=\frac{1}{8 \pi\left(r r_{0}\right)^{1 / 2}} \sum_{n=0}^{\infty}(2 n+1) P_{n}(\cos \gamma) \\
& \cdot \int_{-\infty}^{\infty} \alpha e^{-k \alpha^{2} t} \frac{H_{n+1 / 2}^{(1)}\left(\alpha r_{0}\right)}{U_{n+1 / 2}(\alpha a)}\left\{J_{n+1 / 2}(\alpha r) U_{n+1 / 2}(\alpha a)-H_{n+1 / 2}^{(1)}(\alpha r)\right. \\
& \left.\quad \cdot\left[\alpha \frac{d}{d z} J_{n+1 / 2}(z)+(h-1 /(2 a)) J_{n+1 / 2}(z)\right]_{z=a \alpha}\right\} d \alpha .
\end{aligned}
$$

* This Bulletin, vol. 45 (1939), pp. 310-315. 